\title{
NORMAL CURVATURES AND EULER CLASSES FOR POLYHEDRAL SURFACES IN 4-SPACE
}

\author{
THOMAS F. BANCHOFF
}

\begin{abstract}
Using the approach of singularities of projections into lower dimensional spaces it is possible to define nonintrinsic local curvature quantities at each vertex of a polyhedral surface immersed in 4-space which add up to the normal Euler number of the immersion. Related uniqueness results for lattice polyhedra have been established by B. Yusin.
\end{abstract}

For a surface immersed in Euclidean $n$-space the total (or tangential) curvature can be expressed in terms of singularities of projections into lines, and this interpretation makes it possible to give a unified treatment of curvature for smooth and polyhedral embeddings. In this note we show that for a 2-dimensional surface immersed in 4-space we can carry out a similar construction for the normal curvature in terms of singularities of projections into oriented 3-spaces, recapturing the standard definition for smooth immersions and leading to a new curvature quantity for a polyhedral immersion $f: M^{2} \rightarrow \mathbf{R}^{4}$.

We show how to assign to each vertex $v$ of $u^{2}$ a real number $\nu_{f}(v)$ such that the normal Euler class $\nu(f)$ of the immersion is the sum of the normal curvature at the vertices of $M^{2}$. In contrast to the case of tangential curvatures where the quantities involved are intrinsic, depending only on the metric of the surface in a neighborhood of a vertex, the normal curvature will depend on the immersion $f$. This is to be expected since the (tangential) Euler characteristic is a topological invariant but the normal Euler class depends on the immersion. The constructions depend on the author's paper [2] defining the normal Euler class in terms of singularities of projections.

Working independently, B. Yusin has constructed curvature quantities for vertex stars of lattice polyhedral surfaces in 4-space, with all edges parallel to the four coordinate axes [4]. His values agree with those described in this note and they establish a uniqueness result, showing that the curvature quantities described here are the only ones which can sum to the normal Euler class of an immersed polyhedral surface.

Construction of the curvature quantities. For a smooth immersion $f: M^{2}$ $\rightarrow \mathbf{R}^{4}$ we may define curvature quantities by using moving frames $\left\{e_{1}, e_{2}, e_{3}, e_{4}\right\}$ with $e_{1}, e_{2}$ tangent to $f\left(M^{2}\right)$ and $e_{3}, e_{4}$ normal to the surface. We define the 1forms $\omega_{i j}$ by $d e_{i}=\sum_{j=1}^{4} \omega_{i j} e_{j}$. The tangential curvature of an open set $U$ of $M^{2}$

Received by the editors December 29, 1979 and, in revised form, August 12, 1983.

1980 Mathematics Subject Classification. Primary 57R20, 57Q35, 53C42; Secondary 52A25.

Key words and phrases. Normal curvature, normal Euler class, singularities of projections, curvature for polyhedra, combinatorial formulas for characteristic classes.

(C) 1984 American Mathematical Society $0002-9939 / 84 \$ 1.00+\$ .25$ per pag" 
is defined by

$$
\tau_{f}(U)=\int_{U}-d \omega_{21}
$$

and the normal curvature by

$$
\nu_{f}(U)=\int_{U}-d \omega_{43}
$$

The total tangential curvature $\tau_{f}\left(M^{2}\right)$ is $2 \pi$ multiplied by the (tangential) Euler characteristic $\chi\left(M^{2}\right)$ and the total normal curvature $\nu_{f}\left(M^{2}\right)$ is $2 \pi$ multiplied by the normal Euler number $\nu(f)$ of immersion. The normal Euler number is zero for an embedding of an orientable surface, equal to the negative of the algebraic number of double points for an immersion of an orientable surface, and congruent to $2 \chi\left(M^{2}\right)$ modulo 4 for an embedding of a nonorientable surface. These facts are all proved in the beautiful paper of Whitney [3].

For triangulated surfaces $M^{2}$ and simplex-wise linear immersions $f: M^{2} \rightarrow \mathbf{R}^{4}$ a different approach must be used to define the normal Euler number since we do not have well-defined tangent and normal spaces except at the interior points of 2-dimensional faces. Fortunately it is possible to give a geometric description of $\tau_{f}$ and $\nu_{f}$ which depends on singularities of projections and which applies equally well to smooth and polyhedral surfaces.

Recall that for almost every unit vector $\xi$ in $\mathbf{R}^{4}$ the composition $\xi \circ f: M^{2} \rightarrow$ $\mathbf{R}(\varsigma)$ of $f$ and the orthogonal projection $\xi: \mathbf{R}^{4} \rightarrow \mathbf{R}(\xi)$ to the oriented line along $\xi$ has only nondegenerate critical points. We let $\tau(\xi, f, p)=1$ if $p$ is a maximum or minimum for $\xi \circ f$ and -1 if it is a (nondegenerate) saddle point. If $p$ is not critical for $\xi \circ f$ we set $\tau(\xi, f, p)=0$. The critical point theorem then states that

$$
\sum_{p \in M^{2}} \tau(\xi, f, p)=\chi\left(M^{2}\right) .
$$

We may then integrate this result to get curvature theorems. We may reinterpret $\tau_{f}(U)$ as

$$
\tau_{f}(U)=\frac{1}{C_{3}} \int_{\xi \in S^{3}} \sum_{p \in U} \tau_{f}(\xi, f, p) d \omega_{3},
$$

where $d \omega_{3}$ is the ordinary volume element of $S^{3}$ and $C_{3}=\int_{\xi \in S^{3}} d \omega_{3}$ is the volume of $S^{3}$. Thus $\tau_{f}(U)$ represents the geometric probability that the projection $\xi \circ f$ has a critical point on the open set $U$.

To obtain a corresponding description of the normal Euler number we consider orthogonal projections $\xi^{\perp}: \mathbf{R}^{4} \rightarrow \mathbf{R}^{3}\left(\xi^{\perp}\right)$, where $\mathbf{R}^{3}\left(\xi^{\perp}\right)$ is the oriented hyperplane with outward unit normal vector $\xi$. For almost all $\xi$, the composition $\xi^{\perp} \circ f: M^{2} \rightarrow$ $\mathbf{R}^{3}\left(\xi^{\perp}\right)$ will be an immersion except at a finite number of pinch points, each of which can be given an index $\nu(\xi, f, p)$ which is \pm 1 , with all other points having index 0 . The pinch points correspond to the zeros of the projection of $\xi$ into the tangent planes of $f\left(M^{2}\right)$ and the sum of the indices of these singularities gives the normal Euler number,

$$
\sum_{p \in M^{2}} \nu(\xi, f, p)=\nu_{f}
$$


The number $\nu_{f}$ is independent of the projection $\xi$ used to define it. We may then reinterpret $\nu_{f}(U)$ and the geometric probability that $\xi^{\perp} \circ f$ has a pinch point in $U$, i.e.

$$
\nu_{f}(U)=\frac{1}{C_{3}} \int_{\xi \in S^{3}} \sum_{p \in U} \nu(\xi, f, p) d \omega_{3}
$$

In [1] we generalized the index of singularity $\tau(\xi, f, p)$ so it applies as well to triangulated surfaces and simplex-wise linear mappings. This index will be zero except possibly at vertices $v$ of $M^{2}$ and $\sum_{v \in M} \tau(\xi, f, v)=\chi\left(M^{2}\right)$ as before. The integrated form of this result yields curvature measures at each vertex which turns out to be intrinsic: We define

$$
\tau_{f}(v)=\frac{1}{C^{3}} \int_{\xi \in S^{3}} \tau(\xi, f, v) d \omega_{3}
$$

so $\tau_{f}(U)=\sum_{v \in U} \tau_{f}(v)$.

If we sum over all vertices of $M^{2}$ we obtain a version of the Gauss-Bonnet theorem: $\tau_{f}\left(M^{2}\right)=\chi\left(M^{2}\right)$. We then prove that

$$
\left.\tau_{f}(v)=\frac{1}{2 \pi}\left(2 \pi-\sum_{\Delta^{2} \text { at } v} \text { (interior angle of } \Delta^{2} \text { at } v\right)\right),
$$

an analogue of the Theorem Egregium.

In the case of the normal Euler class, the definition of $\nu_{f}$ depends on pairs of triangles in $M^{2}$ meeting in a vertex $v$. We set

$$
\nu\left(\xi, f, v, \Delta_{i}, \Delta_{j}\right)= \begin{cases}\varepsilon\left(\Delta_{1}, \Delta_{2}\right) & \text { if } \Delta_{1} \cap \Delta_{2}=\{v\} \text { and } \\ & \xi \circ f\left(\Delta_{i}\right) \cap \xi \circ f\left(\Delta_{j}\right) \neq\{\xi \circ f(v)\} \\ 0 & \text { otherwise, }\end{cases}
$$

where $\varepsilon\left(\Delta_{i}, \Delta_{j}\right)= \pm 1$ depending on the orientation on $\mathbf{R}^{4}$ obtained by the orientation of $\Delta_{i}$ followed by that of $\Delta_{j}$. More specifically if the edges at $v$ are arranged in a cyclic order $v w_{1}, v w_{2}, \ldots, v w_{r}$, then $\Delta_{i}$ has vertices $\left\{v, w_{i}, w_{i+1}\right\}$ and $\Delta_{j}$ has vertices $\left\{v, w_{j}, w_{j+1}\right\}$. We set $\varepsilon\left(\Delta_{i}, \Delta_{j}\right)= \pm 1$ depending on whether the frame

$$
\left\{w_{i}-v, w_{i+1}-v, w_{j}-v, w_{j+1}-v\right\}
$$

determines a positive or a negative orientation of $\mathbf{R}^{4}$. (Here $j+1=1$ if $j=r$.)

For a vector $\xi$ such that the projections $\xi^{\perp}\left(v w_{i}\right)$ are in general position in $\mathbf{R}^{3}\left(\xi^{\perp}\right)$, we define

$$
\nu(\xi, f, v)=\sum_{1 \leq i<j \leq r} \nu\left(\xi, f, v, \Delta_{i}, \Delta_{j}\right)
$$

For almost all unit vectors $\xi$ this sum is well defined for all vertices $v$ of $M^{2}$. The number $\nu_{f}=\sum_{v \in M^{2}} \nu(\xi, f, v)$ is independent of the unit vector $\xi$ used to define it. (For a proof of this fact and for an examination of the topological properties of this index, see [2].)

The normal curvature is defined to be

$$
\begin{aligned}
\nu_{f}(U) & =\frac{1}{C_{3}} \int_{\xi \in S^{3}} \sum_{v \in U} \nu(\xi, f, v) d \omega_{3} \\
& =\sum_{v \in U} \frac{1}{C_{3}} \int_{\xi \in S^{3}} \nu(\xi, f, v) d \omega_{3}=\sum_{v \in I} \nu_{f}(v),
\end{aligned}
$$


where

$$
\begin{aligned}
\nu_{f}(v) & =\frac{1}{C_{3}} \int_{\xi \in C^{3}} \nu(\xi, f, v) d \omega_{3} \\
& =\frac{1}{C_{3}} \int_{\xi \in S^{3}} \sum_{1 \leq i<j \leq r} \nu\left(\xi, f, v, \Delta_{i}, \Delta_{j}\right) d \omega_{3} \\
& =\sum_{1 \leq i \leq j \leq r} \frac{1}{C_{3}} \int_{\xi \in S^{3}} \nu\left(\xi, f, v, \Delta_{i}, \Delta_{j}\right) d \omega_{3} .
\end{aligned}
$$

Thus the entire normal Euler number is a sum of contributions at each vertex, each of which is a sum of "generalized angle" expressing the geometric probability that a given pair of triangles $\Delta_{i}$ and $\Delta_{j}$ at $v$ in $\mathbf{R}^{4}$ will intersect at interior points when projected into 3-dimensional subspaces. This generalized angle depends only on the quadruple of unit vectors $\left(a_{i}, a_{i+1}, a_{j}, a_{j+1}\right)$ where

$$
a_{k}\left\|w_{k}-v\right\|=w_{k}-v
$$

for any index $k$. The generalized angle is then a function on the space $F$ of frames of unit vectors in $\mathbf{R}^{4}$. We can then give a combinatorial formula for the normal Euler number by taking the sum of the signed real numbers which represent the geometric probabilities that the projections of a frame have a certain configuration in 3-space.

Can we express these in simpler terms? In certain cases the answer is yes. Yusin has independently considered the normal curvature of polyhedra with all faces parallel to the coordinate planes in $\mathbf{R}^{\mathbf{4}}$. This involves the computation of all expressions $\nu\left( \pm e_{1}, \pm e_{2}, \pm e_{3}, \pm e_{4}\right)$ where the vector $e_{i}$ is a unit vector in the direction of the positive $i$ th axis. Note that there are 16 possible combinations and, for each unit vector $\xi$ not lying in any coordinate plane, there are exactly two combinations for which $\nu\left( \pm \xi, \pm e_{1}, \pm e_{2}, \pm e_{3}, \pm e_{4}\right)$ are nonzero. We thus have $\nu\left(e_{1}, e_{2}, e_{3}, e_{4}\right)=1 / 8$ and $\nu\left(e_{1}, e_{2}, e_{4}, e_{3}\right)=-1 / 8$. All other indices are determined by symmetry. Sums of these indices determine all possible local contributions at vertex stars of lattice polyhedra [4].

\section{BIBLIOGRAPHY}

1. T. Banchoff, Critical points and curvature for embedded polyhedra, J. Differential Geom. 1 (1967), 257-268.

2. $\ldots$, The normal Euler class of a polyhedral surface in 4-space (to appear).

3. H. Whitney, Topology of differentiable manifolds, Lectures in Topology (R. Wilder, ed.), Univ. of Michigan Press, 1941.

4. B. Yusin, A combinatorial formula for the normal Euler class of a lattice 2-manifold in 4-space, Proc. Amer. Math. Soc. 92 (1984), 578-592.

Department of Mathematics, Brown University, Providence, Rhode Island 02912 